\title{
Parameterizations In High Resolution Isopycnal Wind-Driven Ocean Models
}

\author{
Final Report
}

for Period August 1, 1992 through January 31, 1996

\author{
Tommy G. Jensen \\ David A. Randall \\ Principal Investigators
}

Colorado State University

Department of Atmospheric Science

Fort Collins, CO 80523

March 1996

Prepared for

THE U.S. DEPARTMENT OF ENERGY

AGREEMENT NO. DE-FG02-92ER61440

\section{IASTER}




\title{
Parameterizations In High Resolution
}

\section{Isopycnal Wind-Driven Ocean Models}

\author{
Principal Investigators: \\ Tommy G. Jensen \\ David A. Randall \\ Department of Atmospheric Science \\ Colorado State University \\ Fort Collins, Colorado 80523
}

\section{DISCLAIMER}

\begin{abstract}
This report was prepared as an account of work sponsored by an agency of the United States Government. Neither the United States Government nor any agency thereof, nor any of their employees, makes any warranty, express or implied, or assumes any legal liability or responsibility for the accuracy, completeness, or usefulness of any information, apparatus, product, or process disclosed, or represents that its use would not infringe privately owned rights. Reference herein to any specific commercial product, process, or service by trade name, trademark, manufacturer, or otherwise does not necessarily constitute or imply its endorsement, recommendation, or favoring by the United States Government or any agency thereof. The views and opinions of authors expressed herein do not necessarily state or reflect those of the United States Government or any agency thereof.
\end{abstract}




\section{Introduction}

The title of this project is "Parameterizations in High Resolution Isopycnal Wind-Driven Ocean Models” The Principal Investigators are T. G. Jensen and D. A. Randall.

Development of a general quasi-isopycnal code suitable for numerical experimentation and test of parameterizations was the main goal of the proposed research. A versatile ocean modelling system, which we will refer to as the Thermodynamic Ocean Modelling System (TOMS) is the result of this effort.

TOMS is based on an Arbitrary Lagrangian Eulerian (ALE) vertical coordinate and is a hybrid between isopycnal and z-coordinate models. The modelling system is designed for oceanic and coastal flows where the aspect ratio is small.

As part of our development effort, we also proposed to implement and test new numerical schemes, parameterizations of boundary layer flow and development and implementation of mixed layer physics in this model. The additional objectives for the proposed research were:

1. Incorporate various mixed layer parameterizations;

2. implement and test the positive definite Arakawa and Hsu, (1990) scheme;

3. recode a version of the model for a highly parallel architecture;

4. determine effects of various parameterizations of islands, and;

5. determine the correct lateral boundary condition for boundary layer currents, as for instance the Gulf Stream, and other western boundary currents.

Below the work on these topics will be presented and discussed.

\section{Accomplishments}

\subsection{Model Development}

The new model is based on the hydrodynamic FSU Indian Ocean model by Jensen, (1991), but includes prognostic temperature, salinity and tracers as well as mixed layer physics. In addition bottom topography, a simple data assimilation scheme, and a number of new open boundary conditions, which increase the range of possible applications of TOMS, have been included.

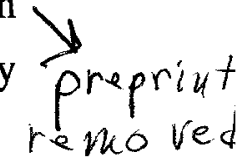

The code consists of 1 main program, 96 subroutines and 33 include files. However, a new model setup is done by changes in the main program and two include files only. The coding is 
Fortran with c-directives to block out unneeded parts. This gives a rather efficient executable, with speeds up to 535 Mflops on a single processor on a Cray YMP C-90. The modular form of the code, as well as the time integration scheme, which is fully explicit, makes implementation of new physical parameterizations very easy. The vertical layer structure is based on an Arbitrary Lagrangian Eulerian coordinate (ALE-method), which makes the model different from the Miami isopycnal model (MICOM) of Bleck and Boudra (1986); Bleck and Smith, (1990), and the Hamburg isopycnal model of Oberhuber, (1993). Basically, we avoid some of the numerical complications, which results from maintaining isopycnals in all layers.
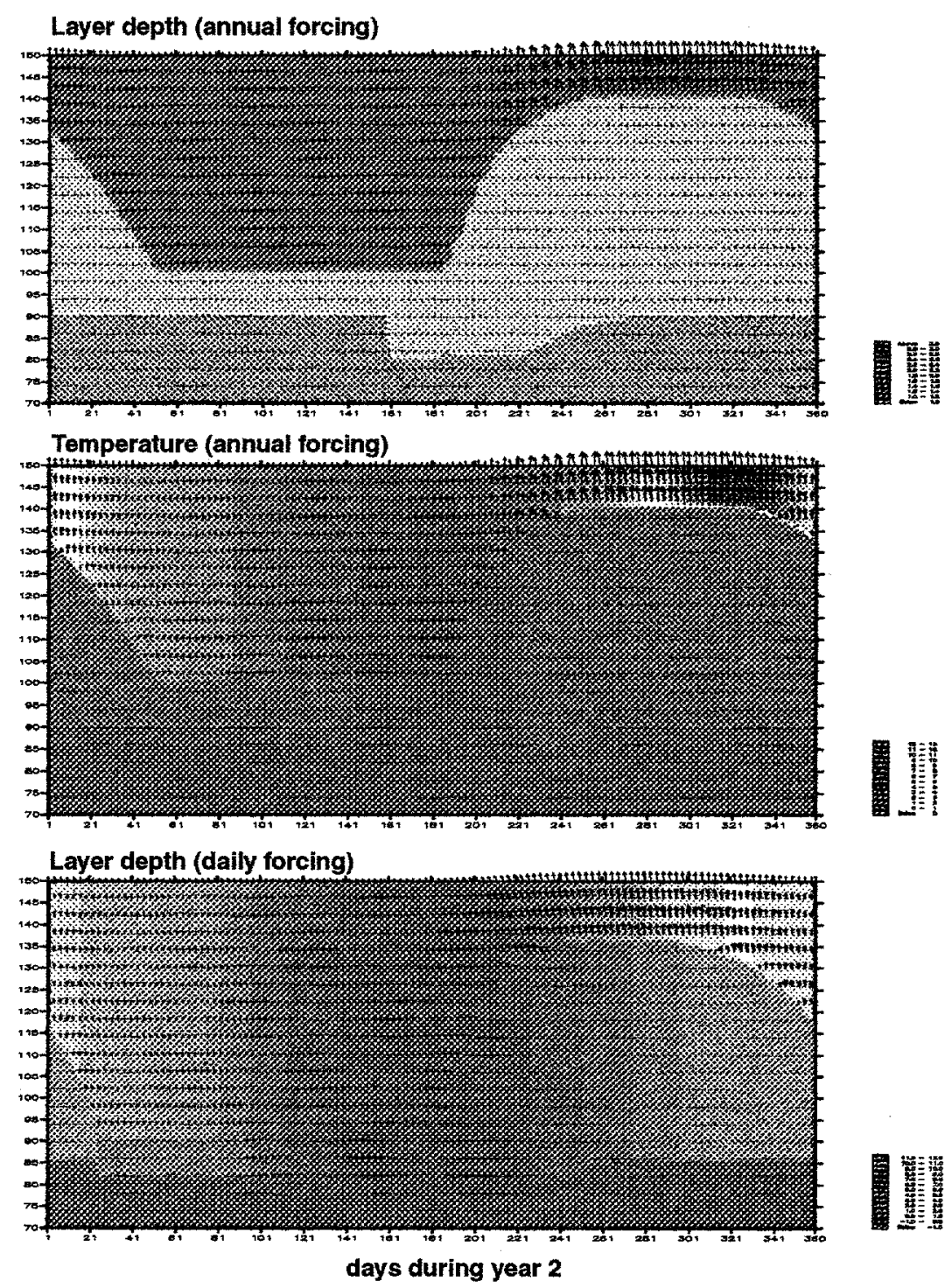

Fig 1. Mixed layer depth evolution in a $150 \mathrm{~m}$ deep three layer model. Note that all layers have the same temperature during the winter. 


\subsection{Mixed layer formulation}

Thermodynamics and mixed layer physics has been fully incorporated in the model as proposed. The prognostic equations for temperature and salinity can be limited to the upper ocean in order to save CPU time. The deeper layers are in that case true isopycnals without mass exchanges. The two uppermost layers have implemented Kraus-Turner mixed layer physics, extended to include exponential damping of TKE, (Oberhuber 1993) and convective overturning. Inclusion of shear induced mixing at the base of all dynamic layers is represented by a Richardson number dependent mixing (Pollard et al., 1973). An entrainment/detrainment formulation similar to that of McCreary and Kundu, $(1988 ; 1989)$ or a diffusion coefficient formulation based on Pacanowski and Philander, (1981) can be chosen. This mixed layer scheme is different than those of Bleck et al, (1989) and Oberhuber (1993), which were designed to restore isopycnals. However, the same basic idea of local balances for TKE is used. The scheme has been tested for equatorial upwelling and downwelling, coastal upwelling and detrainment/entrainment due to heating/ cooling and found to be working satisfactory. Figure 1 shows the layer thickness during the second year of integration from a horizontally uniform 3-layer sea. The forcing was a constant wind stress and an annually varying heat flux, without (center) and with (bottom) a daily cycle. Note that although the sea is fully mixed during the winter, all layers have finite thickness. In this case, the representation is using a $\mathrm{z}$-coordinate. When stratified, the upper layer thickness is determined by the mixed layer physics, for instance being equal to the Monin-Obukhov depth during strong heating. Note that including a daily cycle reduce the sea surface temperature substantially. This is due to convective adjustment during the night.

\subsection{Numerical methods}

\subsubsection{Hsu-Arakawa scheme}

The positive definite advection scheme has been fully implemented as planned, not only for the layer thickness, but also for tracers. The scheme stays positive definite, but can have overshoots as second order schemes. The numerical diffusion is very low compared to the first order donor cell method. For variable values away from zero the scheme behaves very much like the second-order Lax-Wendroff scheme. The only disadvantage is the high computational cost. The scheme is about 10 times more expensive than using a dispersive second-order scheme, e.g. leap-frog and central differences, which is often done for ocean models. However, the present implementation can be better optimized. As an alternative in TOMS, the leap-frog scheme can be chosen with a delimiter on layer thickness. When the layer becomes thinner than a preset limit, the coordinate is gradually changed from Lagrangian to Eulerian. For the upper mixed layer this 
approash work satisfactory with much less computational cost.
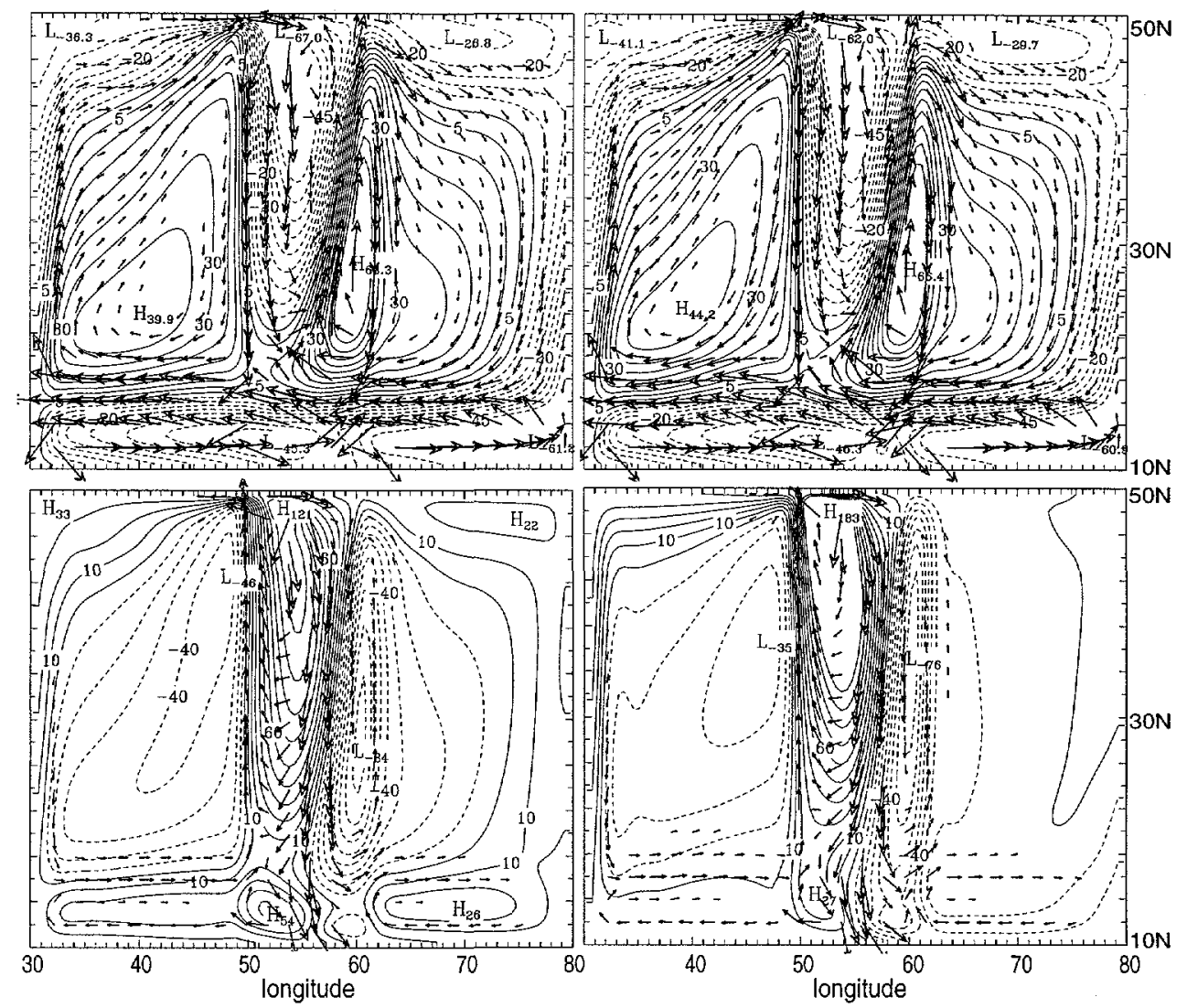

Fig 2. Flow over mid-oceanic ridge and layer thickness anomaly in a 3-layer model, layer 1 (top) and layer 3 (bottom). The solution using the GWR method (right) required only $1 / 16$ of the CPU time from the control (left)

\subsubsection{Gravity Wave Retardation (GWR) Method}

A new method to reduce the speed of the barotropic gravity waves was implemented and tested in the model (Jensen, 1996). The gradient of the surface elevation is reduced by a factor less than one in the momentum equations. This is similar to the method of artificial compressibility used to slow down the speed of sound waves when compressible effects are unimportant, e.g. Chorin, (1967). A factor of about 10 can be gained in CPU time for fully explicit numerical schemes. For a speed up of this order, the method is only sufficiently accurate for large scale ocean circulation where the baroclinic mode dominates the flow. The method does slow down barotropic Rossby waves, energy propagation in particular, so when the dynamics of these waves are 
important, the phase speed of the external gravity waves should only be decreased by a factor 4-5.

Figure 2 shows an example of a mid-latitude ocean with a north-south oriented mid-oceanic ridge. The model was integrated for 1 year without gravity wave retardation as a control and with a GWR factor of $1 / 256$, which slows surface gravity waves by a factor of 16 . Consequently, a factor of 16 can be gained in CPU time. The method is a simpler alternative to implicit methods, which also distort the solution for large Courant numbers.

The GWR method can be applied to spin-up the ocean for decadal simulations of seasonal forced oceanic flow. In the last few years of the simulation, the external gravity wave speed can be gradually increased to minimize errors in the final seasonal circulation. The method can also be used for preliminary investigations where very accurate solutions may not be needed. This makes it possible to simulate a large number of flow scenarios, which otherwise would be prohibitive due to computational costs. Most importantly, the method can be seen as a more general alternative to multi-layer reduced gravity layer models, which, in spite of the lack of the barotropic mode and bottom topography, have been quite successfully used for ocean modelling.

\subsection{Lateral Boundary Conditions}

Since the horizontal resolution in OGCMs is so coarse that the boundary current in most cases is not resolved, it has been argued whether the usual no-slip boundary condition is appropriate. Some investigators imply that a free slip condition is, at least numerically, more consistent.

A paper describing the new thermodynamic ocean model and an application of 'hyperfriction' or 'super drag' to parameterize vorticity transfer along a coastal boundary was presented on the Fifth Symposium on Global Change Studies (Jensen, 1994). In this paper, a simple and very efficient way to increase vorticity transfer from the boundary to the interior flow is described. On the C-grid, the tangential velocity component is not solved directly on the boundary. Traditionally, a ghost point is used on land where the tangential velocity equals that in the ocean in magnitude, but has the opposite sign. By increasing the magnitude (the factor depends

on the grid spacing), one can get the same vorticity transfer as when the boundary layer is resolved. Since the method only requires one additional multiplication per boundary point, this method is extremely efficient, and no additional storage is required.

In the experiment discussed in Jensen (1994), a low-latitude western boundary current crosses the equator and cause instability waves (Fig. 3). We found that in order to get the same 
Resolution $0.2^{\circ} \quad A=750 \mathrm{~m}^{2} / \mathrm{s}$
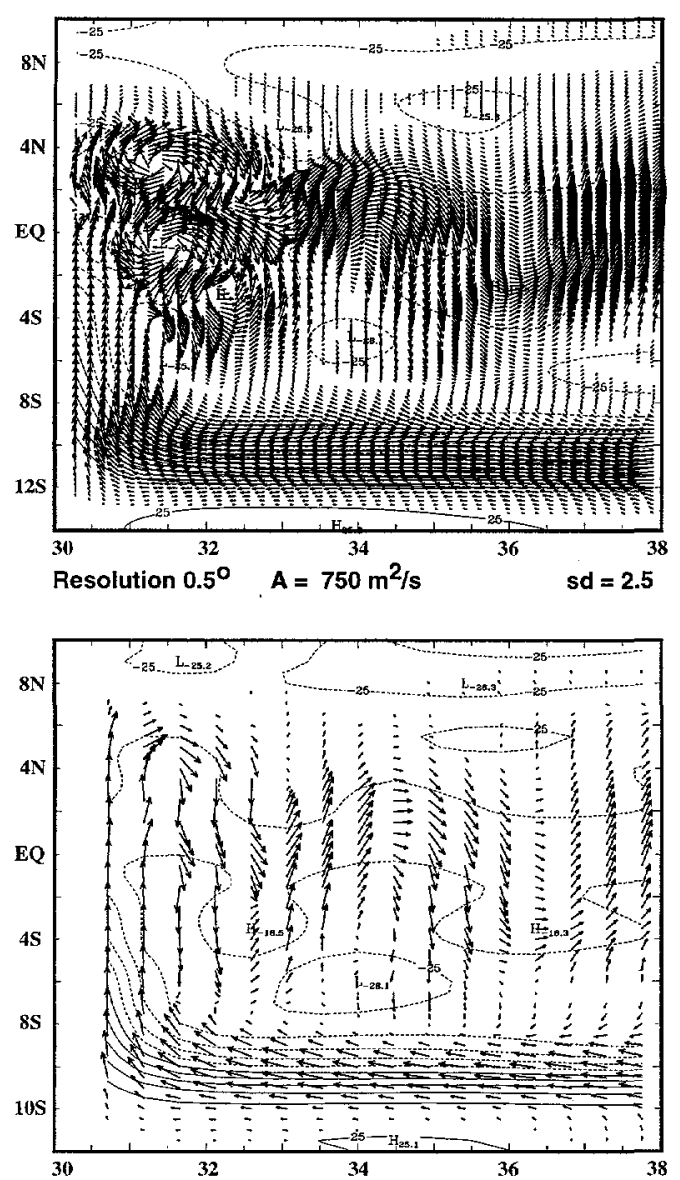

Resolution $0.5^{\circ} \quad A=750 \mathrm{~m}^{2} / \mathrm{s}$
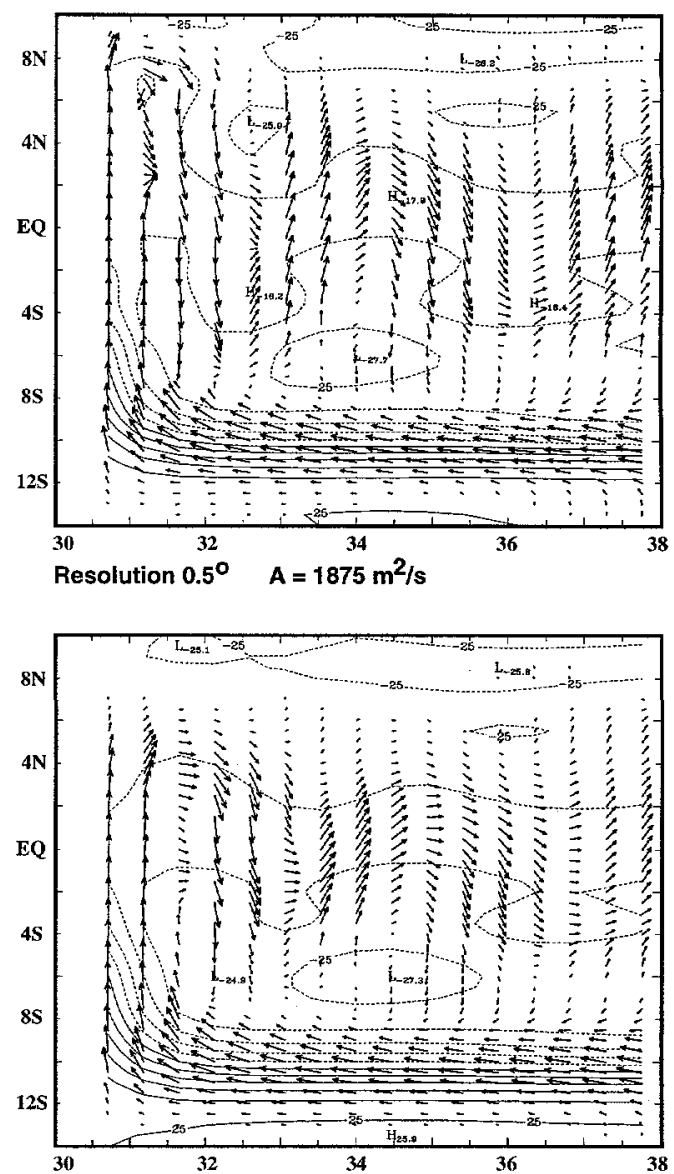

Fig 3. Western boundary current forced by discontinuity in potential energy at $9^{\circ} \mathrm{S}$. Model resolution is $0.1^{\circ}$ (top, left), but only every 4 . vector is shown. Decreasing the resolution increases the latitude of separation (top, right). Increasing lateral friction can restore the correct latitude, but makes the flow more viscous in the interior as well (bottom, right). Using the 'super drag' parameterization (bottom, left) we obtain a very good coarse solution. Vectors for currents larger than $15 \mathrm{~cm} / \mathrm{s}$ are truncated.

separation latitude using a coarse grid, $0.5^{\circ}$ by $0.5^{\circ}$, as in the case where the boundary layer was well resolved $\left(0.1^{\circ}\right.$ by $\left.0.1^{\circ}\right)$, we needed to increase the transfer of vorticity from the boundary to the flow. Traditionally this is done by increasing the lateral friction, i.e. widening the boundary layer. However, it also decreases the amplitude of the instability waves in the interior. As an alternative, we simply increased the vorticity transfer along the boundary using a 'super drag' formulation. It is accomplished using a mirror point with a value equal to $\mathrm{Z}$ times the value for tangential flow component near the boundary. For no slip $Z=-1$, while free slip $Z=0$. For 'super drag', we simply use $\mathrm{Z}<-1$. 
'In most applications harmonic lateral friction is applied with a constant diffusion coefficient. However, in order to prepare the model for other parameterizations, a variable diffusion coefficient can also be specified. Harmonic diffusion can be applied to momentum, layer thickness and all tracers. In addition biharmonic diffusion is implemented for the momentum equations. This is useful to remove $2 \mathrm{dx}$ noise in coarse grid simulations ( $\left.\mathrm{dx}, \mathrm{dy}>0.3^{\circ}\right)$.

\subsubsection{Open Boundary Conditions}

In most applications of numerical ocean models, artificial boundaries are introduced to limit the domain. Along such a boundary we need to apply what is often referred to as an Open Boundary Condition (OBC). A number of local methods used in ocean models have been implemented in TOMS. They are the simple conditions: clamped, prescribed, zero gradient (see Roed and Cooper, 1987); radiation conditions: Camerlengo-O'Brien (1981), Orlanski, (1976); a generalized sponge type condition: Flow Relaxation Scheme (Martinsen and Engedahl, 1987), and a method based on characteristics of the linear equations (Thompson, 1990). These conditions were adapted to layered models using the normal vertical mode projection of Jensen (1991). The OBC's are described in Jensen, (1995b). Data assimilation is also implemented as relaxation towards observations.

\subsection{Parameterization of unresolved geometry}

Many islands and topographic features are too small to be well represented by the model grid. In most oceanic model simulations these features are either enlarged or removed to fit the grid. Our investigations show that a very promising way to represent the effect of unresolved topographic features is to introduce Newtonian friction in the momentum equations, a method which we refer to as the 'wet sand' parameterization. This approach has also been applied successfully in coastal engineering (Danish Hydraulic Institute, personal communication). Figure 4 shows flow around islands in the Indian Ocean. In Fig 4 (bottom), the actual coast lines are used (control). The solution in Fig. 4 (top) is in a box model with increased Newtonian friction used to represent the islands. It is particular appealing that only diagonal terms are involved, so that the terms easily are implemented in implicit models. In TOMS an Euler backward scheme is used. This parameterization has also been successfully used to represent bottom topography.

\subsection{Porting the model to the CM-5}

A data parallel version of a reduced gravity hydrodynamic version of the code was implemented and tested on the Thinking Machine CM-200 and the CM-5. Speeds for a 512 x 256 grid were slightly faster than the computational speed on the Cray YMP. However, the open 


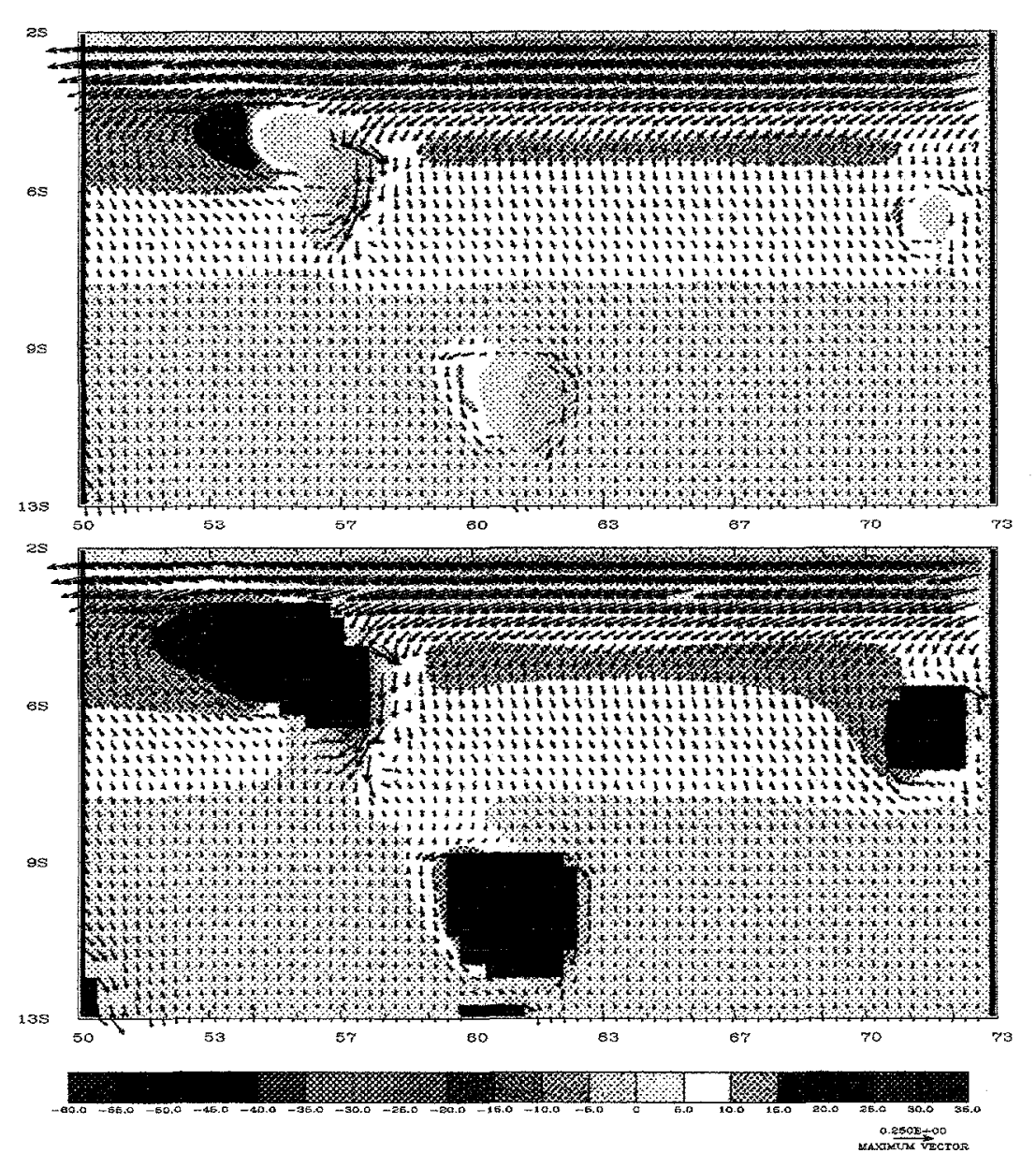

Fig 4. Flow around islands using the 'wet sand' parameterization (top), which apply linear friction to the velocities, and regular land mask (bottom).

boundary conditions used about $40 \%$ of the CPU time. For a closed basin, the data parallel version was significantly faster. The CM-200 version was used on a $256 \times 128$ grid to prove that a semiannual equatorial resonance may exist in the Indian Ocean.

A 21 year run with a 3.5 layer CRAY version of the model, forced by seasonal climatological wind stress, showed that the ratio of semi-annual transports to annual transports along the equator was 2 or larger. However, the ratio of semi-annual to annual zonal wind stress is about 0.5 . The CM-200 version of the model (with one active layer, $1 / 3^{\circ}$ by $1 / 3^{\circ}$ resolution and realistic Indian Ocean coastlines) was used to demonstrate that equatorial Kelvin and Rossby waves may combine to a standing mode with large amplitude when the period of the forcing, $\mathrm{T}$, the internal gravity wave speed, $\mathrm{c}$, and the width, $\mathrm{L}$, of the ocean basin is related through

$$
\mathrm{T}=4 \mathrm{~L} / \mathrm{mc}, \mathrm{m}=1,2,3, \ldots
$$




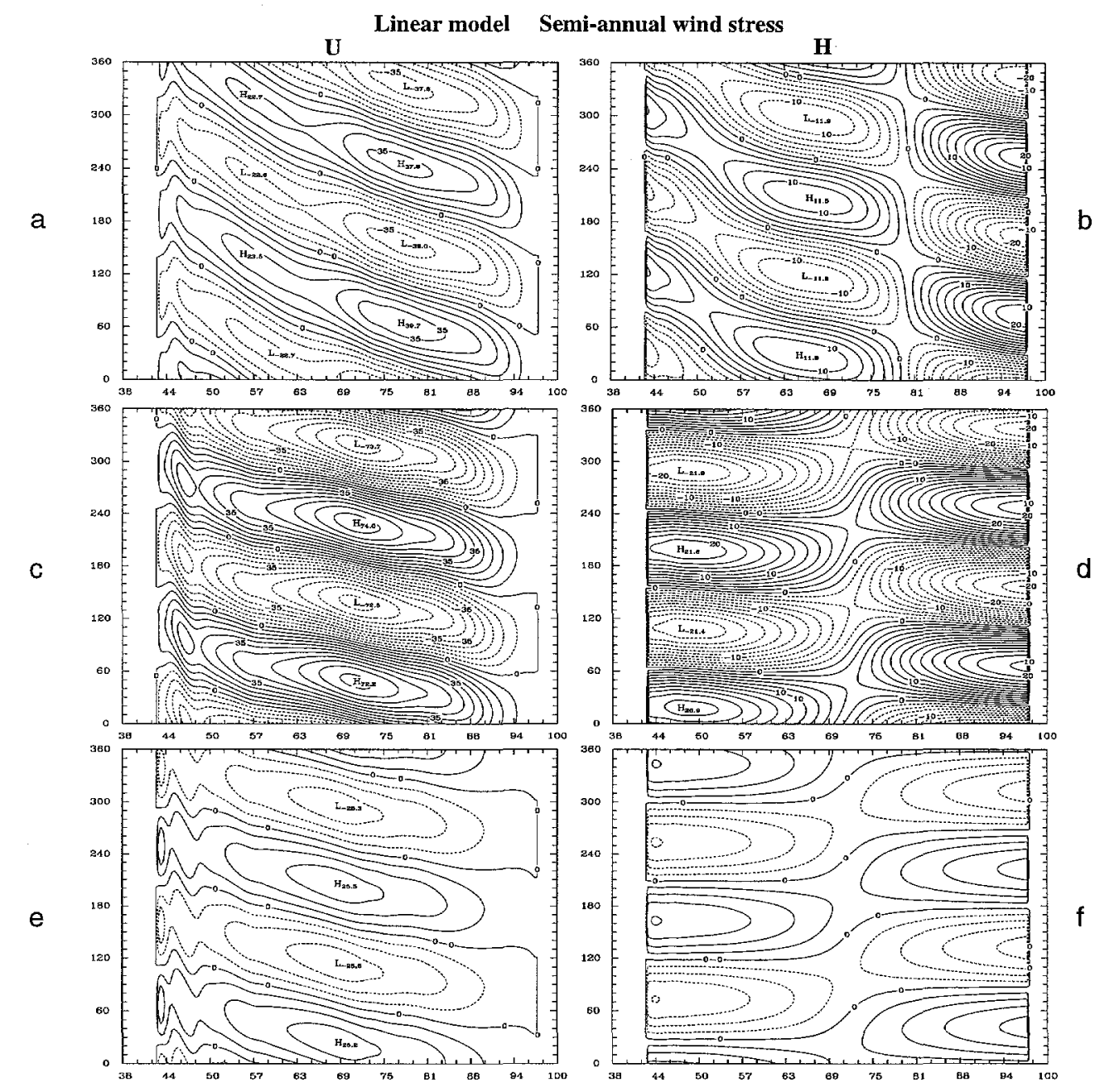

Fig 5. Longitude-time plot of equatorial zonal transport $U$ and upper layer thickness anomaly (in meters) for a linear reduced gravity model of the Indian Ocean. Forcing is semi-annual winds in a $6^{\circ}$ wide band around the equator. The internal gravity wave speed is $1.0 \mathrm{~m} / \mathrm{s}$ ( $\mathrm{a}$ and $\mathrm{b}$ ), $1.4 \mathrm{~m} /$ $\mathrm{s}(\mathrm{c}$ and $\mathrm{d})$ and $2.0 \mathrm{~m} / \mathrm{s}$ (e and $\mathrm{f}$.

Cane and Moore, (1981) had found this relation analytically for a linear model of a rectangular $\beta$-plane ocean, but speculated that the effect would be unlikely to be of importance in an ocean with irregular coastlines, realistic wind forcing and strong non-linear effects. Our study demonstrated that the resonant equatorial basin mode does exist in the Indian Ocean. Figure 5 shows that the equatorial response is strongest for an internal gravity wave speed near $1.4 \mathrm{~m} / \mathrm{s}$ (case $c$ and $d)$. This phase speed is close to that of the second baroclinic mode in a 4-layer model. It was also demonstrated by the CM-200 experiments that non-linearities do not remove this resonance. In fact, for highly non-linear flow, even annual forcing may for semi-annual oscillations. Further details are given (Jensen, (1993). 


\subsection{Model Applications}

We have applied the model to the parameterizations studies mention above and in numerous test cases for model verification. Some of the test cases are given in Jensen (1995)
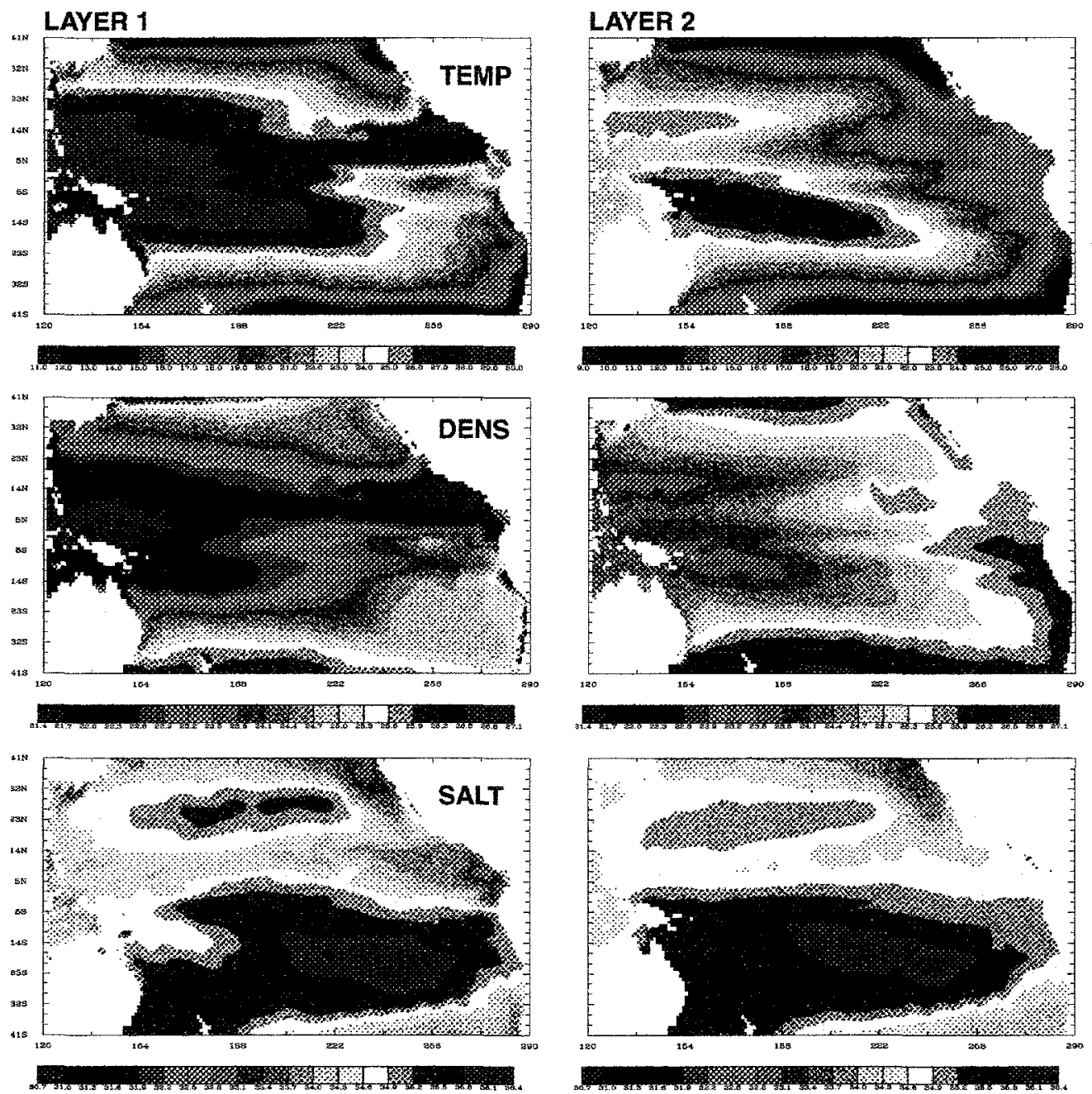

Fig 6. Pacific Model using TOMS. Layer 1 (left) represents the mixed layer depth. It varies between $20 \mathrm{~m}$ and $175 \mathrm{~m}$ in the simulation. Layer 2 varies in thickness from $20 \mathrm{~m}$ to $456 \mathrm{~m}$. The plots show the temperature, density and salinity of the two layers. Layer thickness is not shown.

The latest application is a 2.5 layer upper ocean model for the Pacific Ocean between $40^{\circ} \mathrm{S}$ and $40^{\circ} \mathrm{N}$. The coast lines are resolved to $1^{\circ}$ by $1^{\circ}$, while the model resolution is $0.5^{\circ}$ by $0.5^{\circ}$. This coarse resolution was chosen to facilitate coupling to the CSU atmospheric GCM. The model 
consists of two active layers over an infinite deep third layer. The upper layer, which represents the mixed layer is initially $75 \mathrm{~m}$ thick and layer 2 is $200 \mathrm{~m}$ thick. The model is spun up from rest, forced with annual mean wind stress from a simulation with the CSU GCM. The wind stress was interpolated to the oceanic grid using cubic splines.
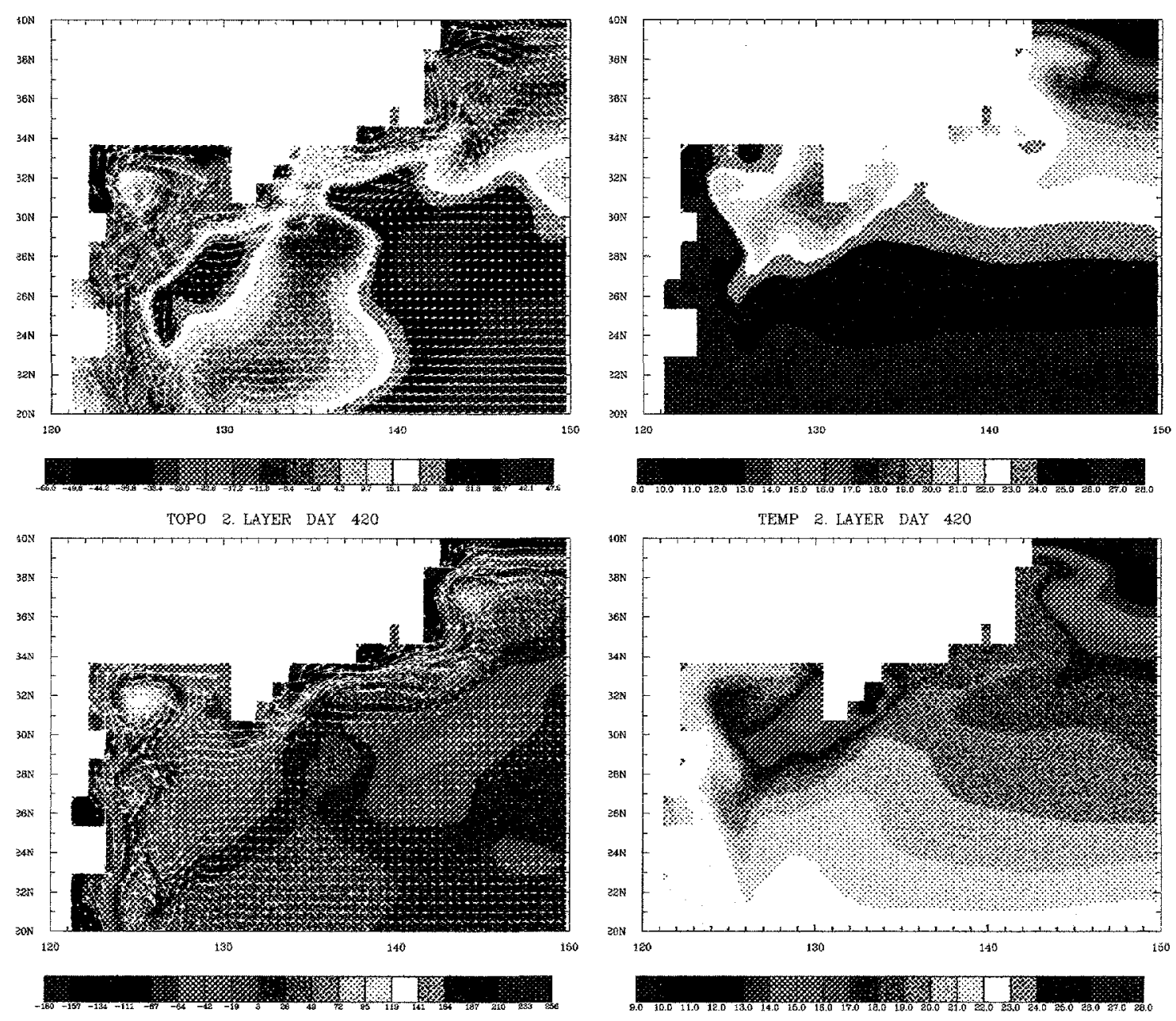

Fig 7.Detailed flow in the model Kuroshio. Layer thickness anomaly (left) and temperature (right) for the mixed layer (top) and second layer (bottom).

The basin ocean model is initialized using the Levitus data sets for temperature and salinity. During the spin-up, the temperature and the salinity is kept constant, e.g. the model is being run in a diagnostic mode. Density is computed using a simplified 2. order polynomial form of the equation of state for sea water (Mamayev, 1976). After the spin up, which for initial testing was one year, fully prognostic equations for temperature and salinity were used. Vertical friction is 
included using the Pacanowski and Philander (1981) formulation, while explicit mixing, e.g. entrainment and detrainment, is modelled using the new mixed layer physics. Shear production of turbulent kinetic energy (TKE) depends on a bulk Richardson number for each layer, while TKE production from the wind in the mixed layer is computed from a modified Kraus-Turner formulation.

Figure 6 shows the temperature, density and salinity fields for the entire domain after 420 days of integration (the last 60 days fully prognostic). The model has at that time set up its equatorward eastern boundary currents, equatorial currents and counter currents and an equatorial undercurrent. Poleward western boundary currents off the coast of Australia and the Kuroshio off the Northeast Asian coast (Fig 7) are also being established. It is obvious from fig. 7 that while the two upper layers have variable temperature (and, in this formulation also density), there are fairly small gradients within each layer compared to the density difference from layer 1 to layer 2 over most of the domain. The deep third layer is kept isopycnal. The main idea here is that waves can propagate across the domain without inducing vertical mixing. In cases of strong vertical shear or strong winds, the model will mix vertically in a way similar to the real ocean.

\subsection{Summary}

We have developed a versatile ocean modelling system for upper ocean simulations. The model is under continuous development and have already been applied to a variety of flows. The current version of TOMS have more features built into the code than we hoped would be possible.

The mixed layer formulation is working as we hoped, although some parameter calibration still need to be done. The main problem, which was coordinate control in the ALE formulation, has been solved. Implementation of the Arakawa-Hsu scheme for tracers was successful, but for many practical problems, it was found to be too costly computationally.

Less successful was the implementation of a data parallel architecture, the CM-200 and CM-5. We succeeded to implement a hydrodynamic version of the code on these machines, but the speed up compared to a single processor on a Cray-YMP was small for the general case with open boundary conditions. However, the code was actually used to produce runs which demonstrated equatorial resonance (Jensen, 1993).

Simple parameterizations of small scale topography such as islands and lateral friction along coast lines were found to work surprising well and are implemented in TOMS. The first report on this work is found in Jensen (1994), but further demonstrations of the method are needed and will be given in the future. 
As part of the development a new numerical method, Gravity Wave Retardation (GWR), was designed and analyzed for layered ocean models. This simple method allows an order of magnitude faster computational speed for an explicit method than that allowed by the CFL criteria. And it is particular appealing that a split between barotropic and baroclinic modes is not needed.

In addition to the applications described above, which were a direct part of the objectives in our proposal, TOMS has been applied to a coastal ocean in a study for open boundary conditions. A numerical technique, the Flow Relaxation Scheme, was implemented and used for general open boundary conditions as well as data assimilation.

Future applications will involve coupling of TOMS to the CSU GCM for ocean basins such as the Pacific. A new project, which involves implementation of the TOMS mixed layer model and geometry parameterizations in the Los Alamos Parallel Ocean Program (POP) model, has also been made possible because of this CHAMMP project.

\section{Publications Supported by this Grant}

Jensen, T. G., 1993: Equatorial Variability and Resonance in a Wind-driven Indian Ocean Model., J. Geophys. Res. - Oceans., 98, 22533-22552.

Jensen, T. G., 1993: Resonant Forcing of the Equatorial Indian Ocean. In: Research Activities in Atmospheric and Oceanic Modelling, G.J. Boer, ed. Rep. 18, WMO/TD No. 533.

Jensen, T. G., 1994: Resonant Equatorial Basin Modes in an Indian Ocean Model.Abstract, AGU Ocean Science Meeting, San Diego, EOS Trans., 75, No. 3,(suppl.)

Jensen, T. G., 1994: Development of a Quasi-Isopycnal Ocean Model. In: Research Activities in Atmospheric and Oceanic Modelling, G.J. Boer, ed. Rep. 19, WMO/TD No. 592.

Jensen, T. G., 1994: A Quasi-Isopycnal Upper Ocean Model for Climate Modelling. Fifth Symposium on Climate Change Studies. American Meteorological Society, Nashville, 77-80

Jensen, T. G., 1995: Description of a Thermodynamic Ocean Modelling System - (TOMS). Technical Report

Jensen, T. G., 1995: Open Boundary Conditions in Stratified Seas. The International Association of the Physical Sciences of the Oceans (IAPSO), Poster Session on 'Coastal Ocean Interaction with the Open Ocean', XXI General Assembly. 
Jensen, T. G., 1995: Open Boundary Conditions in Ocean Models. Technical Report

Jensen, T. G., 1996: Artificial Gravity Wave Retardation in ocean layer models.Abstract, AGU Ocean Science Meeting, San Diego, EOS Trans., 76, No. 3,(suppl.)

Jensen, T. G., 1996: Artificial Retardation of Barotropic Waves in Layered Ocean Models., $J$. Monthly Weather Review, 124, in press.

\section{References}

Arakawa, A. and Y.-J. Hsu, 1990: Energy conserving and potential-enstrophy dissipating schemes for the shallow water equations. Mon. Wea. Rev., 118, 1960-1969.

Bleck, R. and D. B. Boudra, 1986: Wind-driven spin-up in eddy-resolving ocean models formulated in isopycnic and isobaric coordinates,. J. Geophys. Res., 91, 7611-7622

Bleck, R., H. P. Hanson, D. Hu and E. B. Kraus, 1989: Mixed layer-thermocline interaction in a three-dimensional isopycnic coordinate model. J. Phys. Oceanogr., 19, 1417-1439

Bleck, R. and L. T. Smith, 1990: A wind-driven isopycnic coordinate model of the North and Equatorial Atlantic Ocean. 1. Model development and supporting experiments. $J$. Geophys. Res., 95, 3273-3285

Camerlengo, A. L. and J. J. O'Brien, 1980: Open boundary conditions in rotating fluids. J. Comput. Phys., 35, 12-35.

Chorin, A. J., 1967: A numerical method for solving incompressible viscous flow problems. $J$. Comput. Phys., 4, 347-376.

Jensen, T. G., 1991: Modeling the seasonal undercurrents in the Somali Current system. $J$. Geophys Res., 96, 22151-22167.

Jensen, T. G., 1994: A Quasi-Isopycnal Upper Ocean Model for Climate Modelling. Fifth Symposium on Climate Change Studies, American Meteorological Society, Nashville, 77-80.

Jensen, T. G., 1995: Description of a Thermodynamic Ocean Modelling System - (TOMS). Technical Report

Mamayev, O. I., 1975: Temperature-Salinity Analysis of World Ocean Waters. Elsevier, New York. 
Martinsen, E. A., and H. Engedahl, 1987: Implementation and testing of a lateral boundary scheme as an open boundary condition in a barotropic ocean model. Coastal Eng., 11, 603-627.

McCreary, J. P. and P. K. Kundu, 1988: A numerical investigation of the Somali Current during the Southwest Monsoon. J. Mar. Res., 46, 25-58.

McCreary, J. P. and P. K. Kundu, 1989: A numerical investigation of sea surface temperature variability in the Arabian Sea. J. Geophys. Res., 94, 16097-16114.

Oberhuber, J. M., 1993: Simulation of the Atlantic circulation with a coupled sea ice-mixed layer isopycnal general circulation model. Part I: Model description. J. Phys. Oceanogr., 23, 808-829.

Pacanowski, R. C. and G. Philander, 1981: Parameterization of vertical mixing in numerical models of the tropical ocean. J. Phys. Oceanogr., 11, 1443-1451.

Pollard, R. T., P. Rhines and R. O. R. Y. Thompson, 1973: The deepening of the wind-mixed layer. Geophys. Fluid Dyn., 3, 381-404.

Roed, L. P. and C. K. Cooper, 1989: A study of various boundary conditions for wind-forced barotropic numerical ocean models. In: Three-dimensional Models of Marine and Estuarine Dynamics, J. C. J. Nihoul and B. N. Jamart (Eds.), Elsevier, Amsterdam.

Thompson, K. W., 1990: Time-dependent boundary conditions for hyperbolic systems, II. $J$. Comput. Phys., 89, 436-461.

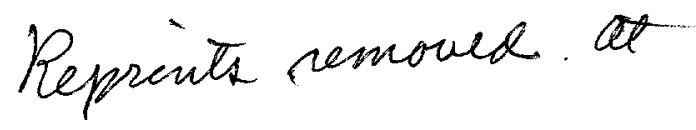

